\title{
Boccioni's ballistics
}

Viewpoints revolve in space and are transformed in time in the art of Umberto Boccioni, a member of the Futurists, who embraced the new sciences and technologies of their age. Boccioni aimed to express a series of relativities.

\section{Martin Kemp}

A new art for a new age. Such was the refrain that ran through avant-garde art, from the time in the nineteenth century when Baudelaire (1821-67) announced his search for a "painter of modern life". By 1900, the "modern life" was being defined by the remorseless spread of the new technologies of energy, transport and communication, and by new notions of time and space

Such new ideas included the non-Euclidean geometry developed by Bernhard Riemann in 1854, and relativity, introduced by Einstein in 1905. The 1907-1912 pictorial revolution of the Cubists Pablo Picasso and Georges Braque involved the dissolution of perspectival space into fractured, interpenetrating planes and plural viewpoints. Retrospectively - and none too convincingly their achievement was hailed as the realization of Riemann's non-Euclidean geometry and even as the embodiment of Einsteinian relativity.

The Futurists in Italy pushed the Cubists' pictorial reforms in the direction of the new sciences and technologies. In "Foundation and Manifesto of Futurism" in 1908, the moustachioed poet F. T. Marinetti belligerently advocated the destruction of museums, the "cemeteries" of art, and lauded the beauty of "a racing car whose hood is adorned with great pipes, like serpents of explosive breath" over the most revered sculptures of antiquity.

Umberto Boccioni, the most creative and versatile of the Futurist artists, was the leading voice behind the "Technical Manifesto" produced by the Futurist painters in 1910 and sole author of their sculptural manifesto two years later. His passionate writings were liberally laced with neologisms, printed in capitals:

\section{UNIVERSAL DYNAMISM, INTERPENETRATION OF PLANES, EXTERIOR and INTERNAL PLASTIC INFINITE, PHYSICAL TRANSCENDENTALISM, INVISIBLE INVOLVING SPACE.}

These alluded, poetically as much as technically, to current mathematics and physics.

His work involved little direct mastery of the technicalities of the new sciences, but rather an intuitive translation of what was sensed to be the crux of developments, not least as translated by the philosopher Henri Bergson. Indeed, Bergson's Creative Evolution (1907) argued that "intelligence", as "the

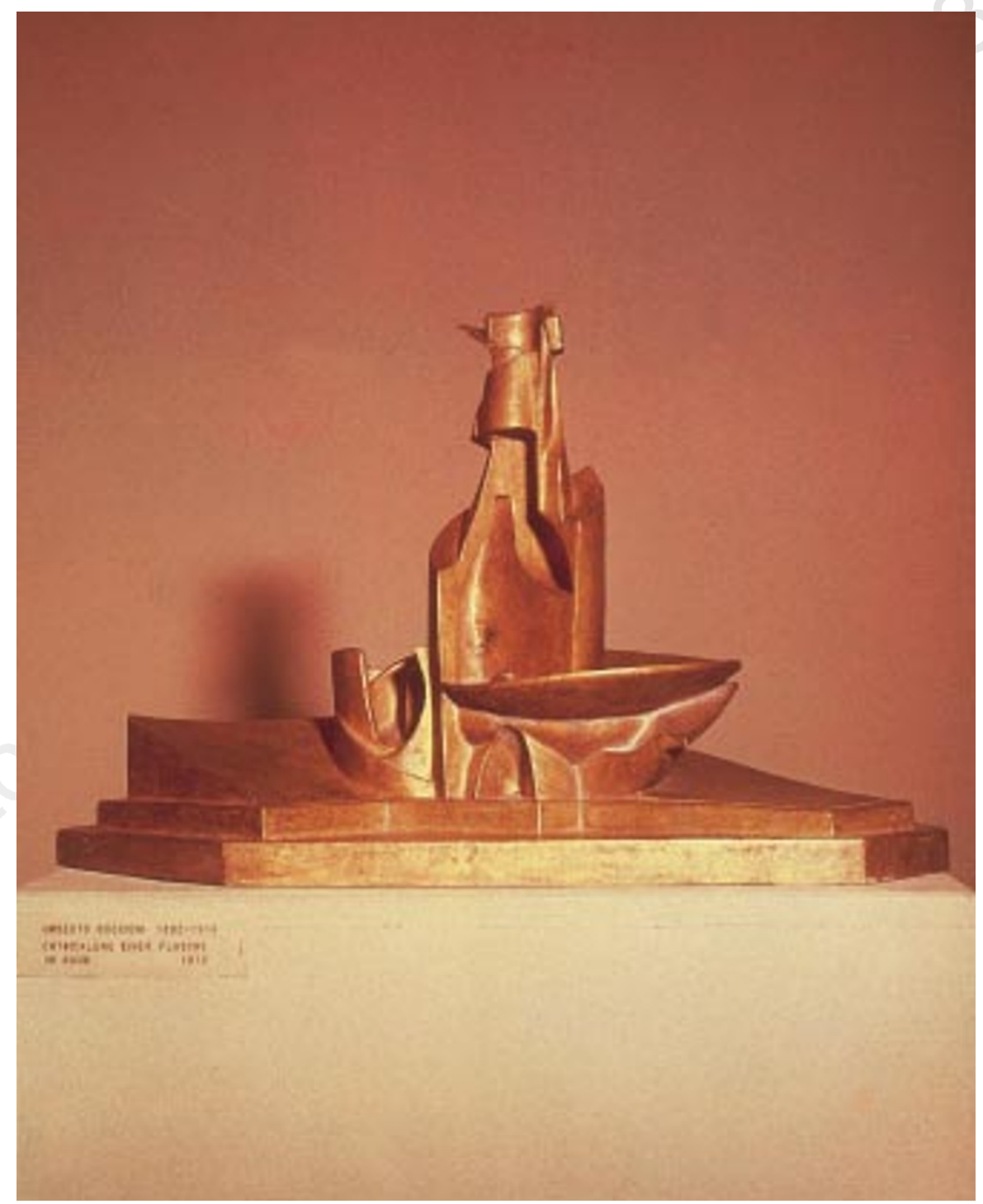

Umberto Boccioni's bronze sculpture Development of a Bottle in Space, 1912, Kunsthaus, Zurich one of various casts of this work.

luminous nucleus" of scientific understanding, must be extended by "instinct" although "instinct, even when enlarged and purified into intuition, forms only a vague nebulosity". Boccioni's emphasis upon "states of mind" — both in his writings and as a title for paintings - clearly signalled that more was involved than scientific logic.

Boccioni strove, in works such as Development of a Bottle in Space, to express a series of relativities - as far as such a thing might be possible in the intractably solid medium of bronze sculpture. We are asked to experience form, space and light as interpenetrating energies rather than as separate physical entities. The spaces occupied by the artist, the work and spectator become interactively fused. Fixed perspective is abandoned in favour of continuously mobile viewpoints, revolving in space and transformed over time.

The Bottle draws space into itself, and makes its own "extension into space, palpable, systematic and plastic".

None of this is 'scientific' in our circumscribed sense, but it does bear witness to Boccioni's determination to use the artists' special toolkit of intellect, imagination, illusion and allusion to forge his own kinds of experiment in the new age.

Martin Kemp is in the Department of the History of Art, University of Oxford, 35 Beaumont Street,

Oxford OX1 2PG, UK. 